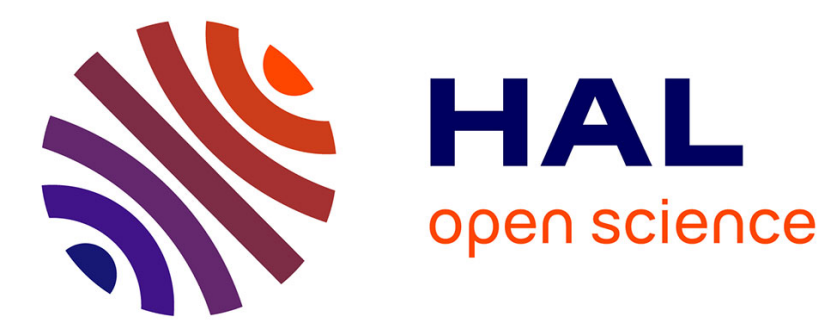

\title{
Stability analysis for systems with saturation and backlash in the loop
}

Sophie Tarbouriech, Isabelle Queinnec, Christophe Prieur

\section{To cite this version:}

Sophie Tarbouriech, Isabelle Queinnec, Christophe Prieur. Stability analysis for systems with saturation and backlash in the loop. CDC 2013 - 52nd IEEE Conference on Decision and Control, Dec 2013, Florence, Italy. pp.6652-6657. hal-00926128

\section{HAL Id: hal-00926128 \\ https://hal.science/hal-00926128}

Submitted on 9 Jan 2014

HAL is a multi-disciplinary open access archive for the deposit and dissemination of scientific research documents, whether they are published or not. The documents may come from teaching and research institutions in France or abroad, or from public or private research centers.
L'archive ouverte pluridisciplinaire HAL, est destinée au dépôt et à la diffusion de documents scientifiques de niveau recherche, publiés ou non, émanant des établissements d'enseignement et de recherche français ou étrangers, des laboratoires publics ou privés. 


\title{
Stability analysis for systems with saturation and backlash in the loop
}

\author{
Sophie Tarbouriech, Isabelle Queinnec, Christophe Prieur
}

\begin{abstract}
This paper deals with the stability analysis problem for linear systems with saturation and backlash in the loop. The resulting system controlled by a static output feedback is a dynamical model with nested backlash and saturation operators. Uniform ultimate boundedness stability is tackled in a regional (local) or global context depending on the stability property of the open-loop system. Suitable regions of the state space in which the closed-loop trajectories can be captured are characterized, together with estimates of the basin of attraction of such regions. Convex optimization problems are proposed in order to give a constructive solution (in the sense of theoretical conditions associated to numerical procedures).
\end{abstract}

\section{INTRODUCTION}

Physical control systems are often subject to magnitude limitation in the input and/or the output. This type of nonlinearity may drastically reduce the performance of the closed-loop system or even lead to instability. Therefore, for these reasons the stability analysis or stabilization problems of control systems with saturation in the input attracted research efforts for several decades (see, e.g., [8], [6], [14]). Another common nonlinearity that limits performance for many applications is the backlash. These operators are nonlinear elements with memory. They are usual phenomena in many physical systems, such as electrical inductors, piezoactuators, gear trains and mechanical friction systems (see e.g. the survey [10] on such memory elements and other operators as Krasnolsel'skii-Pokrovskii hysteresis). They still constitute an open source of both theoretical and practical problems [11], [1].

Few papers have considered both nonlinearities in view of stability analysis or control design purpose. One can cite [4] in which the authors study the presence of saturation and backlash in series at the input of the system. The technique proposes to inverse the backlash in order to cancel its undesirable effects. Then the resulting system corresponds to a system with a new saturation for which the level is depending on the magnitude constraint of the previous saturation and on the parameters of the backlash. The current paper has chosen another route and then does not search for inverting the backlash element. Stability analysis results are studied from the use of Lyapunov theory arguments. More precisely, generalized sector conditions using properties of the saturation and backlash operators, associated to Lyapunov arguments allow us to analyze the stability in a regional (local) or global

S. Tarbouriech and I. Queinnec are with CNRS, LAAS, 7 avenue du colonel Roche, F-31400 Toulouse, France and Univ de Toulouse, LAAS, F31400 Toulouse, France. tarbour@laas.fr, queinnec@laas.fr

C. Prieur is with Gipsa-lab, Department of Automatic Control, Grenoble Campus, 11 rue des Mathématiques, BP 46, 38402 Saint Martin d'Hères, France christophe.prieuregipsa-lab.fr context. The regional result (see Theorem 1 below), presents sufficient conditions to prove that a compact set (inner set) is a finite-time attractor, for all admissible initial conditions belonging to another compact set (outer set). Then, the second contribution of the paper (see Theorem 2) adapts the technique of Theorem 1 to the global case. In this case, the outer set becomes all the state space, provided that the openloop system is not strictly exponentially unstable. Furthermore, at the opposite of conditions developed in [17], we do not need to use the time-derivative version of the system. Moreover, while [7] addresses the characterization of the global finite-time attractor (no saturation being considered), our approach uses the knowledge of the Lyapunov function and, in this sense, can be considered as bringing a new brick to complement the method proposed in [7]. Both in the local and global cases, the conditions are given in terms of bilinear matrix inequalities and associated algorithms based on LMI constraints are proposed to numerically implement the theoretical conditions.

Notation. For two vectors $x, y$ of $\Re^{n}$, the notation $x \succeq y$ means that $x_{(i)}-y_{(i)} \geq 0, \forall i=1, \ldots, n . \mathbf{1}$ and $\mathbf{0}$ denote the identity matrix and the null matrix of appropriate dimensions, respectively. $x \in \Re_{+}^{n}$ means that $x \succeq \mathbf{0}$. The Euclidian norm is denoted $\|\cdot\| . A^{\prime}$ and $\operatorname{trace}(A)$ denote the transpose and the trace of $A$, respectively. For two symmetric matrices, $A$ and $B, A>B$ means that $A-B$ is positive definite. In partitioned symmetric matrices, the symbol $\star$ stands for symmetric blocks. $\lambda_{\max }(A)$ (respectively, $\lambda_{\min }(A)$ ) denotes the maximal (respectively, minimal) eigenvalue of the matrix $A$.

\section{Problem Formulation}

The class of systems under consideration consists of systems with saturation and backlash in the loop. The system is described by:

$$
\begin{aligned}
\dot{x} & =A x+B u \\
y & =C x+D \Phi[w] \\
w & =E x \\
u & =\operatorname{sat}(K y)
\end{aligned}
$$

where $x \in \Re^{n}$ is the state, $u \in \Re^{m}$ is the input of the plant, $y \in \Re^{p}$ is the measured output of the plant and $w \in \Re^{q}$ is the input of the nonlinearity $\Phi . A, B, C, D, E$ and $K$ are known matrices of appropriate dimensions. sat denotes the classical vector-valued saturation function defined as $\forall i=1, \ldots, m$ : $(\operatorname{sat}(z))_{(i)}=\operatorname{sat}\left(z_{(i)}\right)=\operatorname{sign}\left(z_{(i)}\right) \min \left(u_{0(i)},\left|z_{(i)}\right|\right)$ with $u_{0(i)}>0$ the $i$ th level of the saturation. $\Phi$ is a componentwise backlash operator (see, for example, [10], [12], [20], [2]). We denote the set of continuous, piecewise differentiable functions $f:[0,+\infty) \rightarrow \Re^{q}$ by $\mathcal{C}_{p w}^{1}\left([0,+\infty) ; \Re^{q}\right)$, 
that is the set of continuous functions $w$ being, for some unbounded sequence $\left(t_{j}\right)_{j=0}^{\infty}$ in $[0,+\infty)$ with $t_{0}=0$, continuously differentiable on $\left(t_{j-1}, t_{j}\right)$ for all $j \in \mathbb{N}$. Given the vector $\rho$ in $\Re_{+}^{q}$ and $L=\operatorname{diag}\left(\ell_{(i)}\right)$, with positive values $\ell_{(i)}, i=1, \ldots, q$, the operator $\Phi$ is defined as follows, for all $f \in \mathcal{C}_{p w}^{1}\left([0,+\infty) ; \Re^{q}\right)$, for all $j \in \mathbb{N}$, for all $t \in\left(t_{j-1}, t_{j}\right)$ and for all $i \in\{1, \ldots, q\}$ :

$$
(\overbrace{\Phi[f]}(t))_{(i)}=\left\{\begin{array}{l}
\ell_{(i)} \dot{f}_{(i)}(t) \text { if } \dot{f}_{(i)}(t) \geq 0 \\
\text { and }(\Phi[f](t))_{(i)}=\ell_{(i)}\left(f_{(i)}(t)-\rho_{(i)}\right) \\
\ell_{(i)} \dot{f}_{(i)}(t) \text { if } \dot{f}_{(i)}(t) \leq 0 \\
\text { and }(\Phi[f](t))_{(i)}=\ell_{(i)}\left(f_{(i)}(t)+\rho_{(i)}\right) \\
0 \text { otherwise }
\end{array}\right.
$$

where $0=t_{0}<t_{1}<\ldots$ is a partition of $[0,+\infty)$ such that $f$ is continuously differentiable on each of the intervals $\left(t_{j-1}, t_{j}\right), j \in \mathbb{N}$. Thus, $\Phi$ is a time-invariant nonlinearity with slope restriction, as in [13]. Note however that it is a memory-based operator, since to compute it, we need to have information about the past values of $w$ (this is not the case in [13]).

Throughout the paper, we define the set of admissible initial conditions $(w(t=0))$, from which we want to guarantee the stability, as follows

$$
L(w(0)+\rho) \succeq \Phi[w](0) \succeq L(w(0)-\rho)
$$

According to [11], [3], that means that the nonlinearity $\Phi$ is active. Then, with (2), one gets

$$
L(w(t)+\rho) \succeq \Phi[w](t) \succeq L(w(t)-\rho), \forall t \geq 0
$$

For conciseness, throughout the paper, we denote $\dot{\Phi}$ instead of $\overbrace{\Phi[w]}$, and $\Phi$ instead of $\Phi[w]$. Let us define the nonlinearities $\varphi$ and $\Psi$

$$
\begin{aligned}
& \varphi=\operatorname{sat}(K y)-K y \\
& \Psi=\Phi-L w=\Phi-L E x
\end{aligned}
$$

Hence, the closed-loop system (1) reads:

$$
\begin{aligned}
\dot{x} & =(A+B K(C+D L E)) x+B \varphi+B K D \Psi \\
w & =E x
\end{aligned}
$$

It is important to note that both nonlinearities are nested since, by definition of $y, \varphi$ depends on $\Psi$ :

$$
\varphi=\operatorname{sat}\left(K_{0} x+K D \Psi\right)-\left(K_{0} x+K D \Psi\right)
$$

with $K_{0}=K(C+D L E)$.

The presence of the backlash operator $\Phi$ may induce the existence of multiple equilibrium points or a limit cycle around the origin. Furthermore, in a neighborhood of the origin, system (1) operates in open loop. In parallel, the presence of the saturation asks for characterizing suitable regions of the state space in which the stability of the closedloop saturated system can be ensured [14]. The regional (local) uniform ultimate boundedness stability (see [9, Section 4.8]) of the system is then carried out. Hence, the proposed approach aims at characterizing two sets such that the closedloop trajectories initialized in the outer set are ultimately bounded in the inner set. It is important to emphasize that the proposed technique does not require the open-loop system to be stable. Nevertheless, depending on the open-loop stability, the global uniform boundedness stability context is also carried out. In this case, the outer set corresponds to the whole state space. This is stated in the following problems:

Problem 1 (Regional Case): Considering $A_{0}=A+$ $B K(C+D L E)=A+B K_{0}$ being Hurwitz, characterize the regions $\mathcal{S}_{1}$ and $\mathcal{S}_{0}$ of the state space such that the closed-loop trajectories of the system (7) remain confined in $\mathcal{S}_{1}$ and are uniformly ultimately bounded in the set $\mathcal{S}_{0}$, when initialized as in (3).

Imposing $A_{0}=A+B K_{0}$ Hurwitz is classical since it corresponds to system (1) when the saturation and the backlash $\Phi$ are neglected. That corresponds to consider $u=K y$ and to replace $\Phi$ by $L w$ (which corresponds to take $\varphi=\mathbf{0}$ and $\Psi=\mathbf{0}$ in system (7)).

Problem 2 (Global Case): Assume that $A$ is Hurwitz. Considering $A_{0}=A+B K(C+D L E)=A+B K_{0}$ being Hurwitz, characterize the region $\mathcal{S}_{0}$ of the state space in which the solutions of system (1) are uniformly ultimately bounded when initialized as in (3).

\section{MAIN RESUlTS}

\section{A. Preliminary results}

From Lemma 3.1 in [16] and (4), we can formulate the following properties with respect to $\Psi$.

Lemma 1: For any diagonal positive definite matrices $N_{1}$, $N_{2}, N_{3}$ in $\Re^{m \times m}$, with $N_{3} \geq \mathbf{1}$, we have, for all $w \in$ $\mathcal{C}_{p w}^{1}\left([0,+\infty) ; \Re^{q}\right)$, for all $t \in\left(t_{i-1}, t_{i}\right)$

$$
\begin{aligned}
&(\dot{\Psi}+L \dot{w})^{\prime} N_{1} \Psi \leq 0, \\
&(\dot{\Psi}+L \dot{w})^{\prime} N_{2}\left(\dot{\Psi}+\left(\mathbf{1}-N_{3}\right) L \dot{w}\right) \leq 0, \\
&-L \rho \preceq \Psi \preceq L \rho
\end{aligned}
$$

where $0=t_{0}<t_{1}<\ldots$ is a partition of $[0,+\infty)$ such that $w$ is continuously differentiable on each of the intervals $\left(t_{j-1}, t_{j}\right), j \in \mathbb{N}$.

\section{B. Stability analysis}

The following result relative to Problem 1 can be given.

Theorem 1: Consider $A_{0}=A+B K(C+D L E)=$ $A+B K_{0}$ being Hurwitz. If there exist a symmetric positive definite matrix $P \in \Re^{n \times n}$, four diagonal positive definite matrices $N_{1} \in \Re^{q \times q}, N_{2} \in \Re^{q \times q}, T_{3} \in \Re^{q \times q}$ and $T \in$ $\Re^{m \times m}$, a matrix $G \in \Re^{m \times n}$, two positive scalars $\eta, \tau_{1}$ satisfying the following conditions

$$
\begin{aligned}
& M_{1}<\mathbf{0} \\
& \rho^{\prime} L T_{3} L \rho-\tau_{1} \leq 0 \\
& \left(\begin{array}{cc}
P & G_{(i)}^{\prime} \\
\star & \eta u_{0(i)}^{2}
\end{array}\right) \geq \mathbf{0}, i=1, \ldots ., m \\
& \eta<1
\end{aligned}
$$

with $M_{1}$ defined in (13) (see at the top of the next page), then, for any initial admissible conditions $(x(0), \Psi(0))$, with 


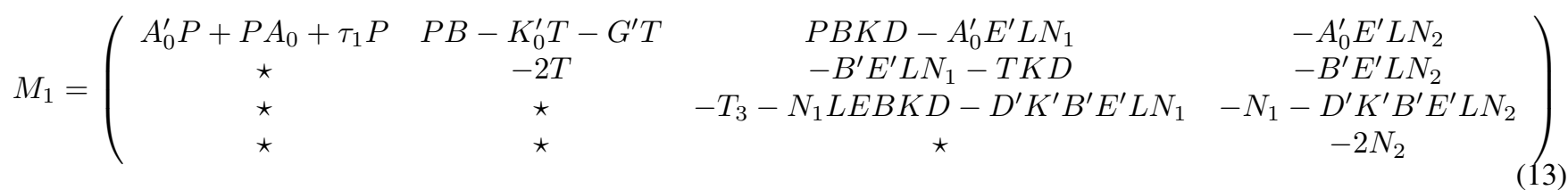

$x(0) \in \mathcal{S}_{1}$, the resulting trajectories of the closed-loop system (1) are uniformly ultimately bounded in the set $\mathcal{S}_{0}$, where the sets $\mathcal{S}_{1}$ and $\mathcal{S}_{0}$ are defined as follows:

$$
\begin{aligned}
& \mathcal{S}_{1}=\left\{x \in \Re^{n} ; x^{\prime} P x \leq \eta^{-1}\right\} \\
& \mathcal{S}_{0}=\left\{x \in \Re^{n} ; x^{\prime} P x \leq 1\right\}
\end{aligned}
$$

Proof: Consider a quadratic Lyapunov function candidate $V$ defined by $V(x)=x^{\prime} P x, P=P^{\prime}>\mathbf{0}$, for all $x$ in $\Re^{n}$. Regarding the nonlinearity $\varphi$, the proof relies on the application of the generalized sector condition [15] stating that for any diagonal positive define matrix $T$, one satisfies: $-2 \varphi^{\prime} T(\operatorname{sat}(K y)+G x) \geq 0$ for any $x \in S\left(G, u_{0}\right)=\{x \in$ $\left.\Re^{n} ;-u_{0(i)} \leq G_{(i)} x \leq u_{0(i)}, i=1, \ldots, m\right\}$. Furthermore, the satisfaction of relation (15) ensures that the set $\mathcal{S}_{1}$, defined in (17) is included in the region $S\left(G, u_{0}\right)$ in which the sector condition on $\varphi$ is satisfied. Hence, in $\mathcal{S}_{1}$, we can write $\dot{V}(x) \leq \dot{V}(x)-2 \varphi^{\prime} T(\operatorname{sat}(K y)+G x)$. Note that the satisfaction of relation (16) guarantees that $\mathcal{S}_{1}$ contains $\mathcal{S}_{0}$.

We want to verify that there exists a class $\mathcal{K}$ function $\alpha$ such that $\dot{V}(x) \leq-\alpha(V(x))$, for all $x$ such that $x^{\prime} P x \geq$ 1 and $x^{\prime} P x \leq \eta^{-1}$ (i.e. for any $x \in \mathcal{S}_{1} \backslash \mathcal{S}_{0}$ ), and for all nonlinearities $\Psi$ satisfying Lemma 1 (i.e. satisfying relations (9), (10), (11)).

By using the S-procedure, it is sufficient to check that $\mathcal{L}<0$, where

$$
\begin{aligned}
\mathcal{L}= & \dot{V}(x)-\tau_{1}\left(1-x^{\prime} P x\right) \\
& -\Psi^{\prime} T_{3} \Psi+\rho^{\prime} L T_{3} L \rho-2(\dot{\Psi}+L \dot{w})^{\prime} N_{1} \Psi \\
& -2(\dot{\Psi}+L \dot{w})^{\prime} N_{2}\left(\dot{\Psi}+\left(\mathbf{1}-N_{3}\right) L \dot{w}\right) \\
& -2 \varphi^{\prime} T(\operatorname{sat}(K y)+G x)
\end{aligned}
$$

with $\tau_{1}$ a positive scalar and $T_{3}$ a positive diagonal matrix. Choosing $N_{3}=1$, noting that $\dot{w}=E A_{0} x+E B \varphi+$ $E B K D \Psi$, from the definitions of $\varphi$ and of $\Psi$ in (8) and in (6), noting that $\dot{V}(x)=x^{\prime}\left(A_{0}^{\prime} P+P A_{0}\right) x+2 x^{\prime} P B \varphi+$ $2 x^{\prime} P B K D \Psi$, it follows that $\mathcal{L}=\mathcal{L}_{0}+\rho^{\prime} L T_{3} L \rho-\tau_{1}$ with

$$
\mathcal{L}_{0}=\left(\begin{array}{c}
x \\
\varphi \\
\Psi \\
\dot{\Psi}
\end{array}\right)^{\prime} M_{1}\left(\begin{array}{c}
x \\
\varphi \\
\Psi \\
\dot{\Psi}
\end{array}\right)
$$

and $M_{1}$ defined in (13). The satisfaction of relations (12) and (14) implies both $\mathcal{L}_{0}<0$ and $\rho^{\prime} L T_{3} L \rho-\tau_{1} \leq 0$, and then $\mathcal{L}<0$, for all $(x, \varphi, \Psi, \dot{\Psi}) \neq \mathbf{0}$.

Therefore, the satisfaction of relations (12)-(16) ensures that there exists $\varepsilon>0$, such that $\mathcal{L} \leq-\varepsilon\left\|\left(x^{\prime} \varphi^{\prime} \Psi^{\prime} \dot{\Psi}^{\prime}\right)^{\prime}\right\|^{2} \leq$ $-\varepsilon x^{\prime} x$. Hence, since by definition one gets $\dot{V}(x) \leq \dot{V}(x)-$ $\tau_{1}\left(1-x^{\prime} P x\right) \leq \mathcal{L}$, one can also verify

$$
\dot{V}(x) \leq-\varepsilon x^{\prime} x, \forall x \text { such that } \eta^{-1} \geq x^{\prime} P x \geq 1
$$

Consider now a solution of (1) starting from any admissible initial condition at $t_{0}$ such that $\eta^{-1} \geq x\left(t_{0}\right)^{\prime} P x\left(t_{0}\right) \geq 1$. According to (20), there exists a time $T \geq t_{0}+\left(x\left(t_{0}\right)^{\prime} P x\left(t_{0}\right)-\right.$ 1) $\lambda_{\max }(P) / \epsilon$ such that $x(t) \in \mathcal{S}_{1}, \forall t \geq T$. Furthermore, $\mathcal{S}_{1}$ is an invariant set for the trajectories of system (1). Hence, in accordance with [9], it follows that the trajectories are uniformly ultimately bounded in $\mathcal{S}_{0}$. That concludes the proof of Theorem 1.

Using the same tools and assuming that the matrix $A$ is Hurwitz we can propose the following solution to the global case, i.e., to Problem 2.

Theorem 2: Assume that $A$ is Hurwitz. Consider $A_{0}=$ $A+B K(C+D L E)=A+B K_{0}$ being Hurwitz. A positive scalar $\tau_{1}$ being given, if there exist a symmetric positive definite matrix $P \in \Re^{n \times n}$, four diagonal positive definite matrices $N_{1} \in \Re^{q \times q}, N_{2} \in \Re^{q \times q}, T_{3} \in \Re^{q \times q}$ and $T \in$ $\Re^{m \times m}$ satisfying the following LMI conditions

$$
\begin{gathered}
M_{2}<\mathbf{0} \\
\rho^{\prime} L T_{3} L \rho-\tau_{1} \leq 0
\end{gathered}
$$

with $M_{2}$ defined in (22) (see at the top of the next page), then, for any initial admissible conditions $(x(0), \Psi(0))$, the resulting trajectories of the closed-loop system (1) are uniformly ultimately bounded in the set $\mathcal{S}_{0}$ defined as follows

$$
\mathcal{S}_{0}=\left\{x \in \Re^{n} ; x^{\prime} P x \leq 1\right\}
$$

Proof: The proof follows the same stages as in that one of Theorem 1. In this case one wants to verify that there exists a class $\mathcal{K}$ function $\alpha$ such that $\dot{V}(x) \leq-\alpha(V(x))$, for all $x$ such that $x^{\prime} P x \geq 1$ (i.e. for any $x \in \Re^{n} \backslash \mathcal{S}_{0}$ ), for all nonlinearities $\Psi$ satisfying Lemma 1 (i.e. satisfying relations (9), (10), (11)) and any $\varphi$ satisfying $-2 \varphi^{\prime} T \operatorname{sat}(K y) \geq 0$, $\forall x$. In the global context, one then chooses $G=\mathbf{0}$.

Theorem 1 guarantees the finite-time convergence of the solutions to system (1) inside $\mathcal{S}_{0}$ for any initial condition satisfying (3) and belonging to $\mathcal{S}_{1}$. Therefore the property is locally satisfied. Theorem 2 assures the same type of convergence but in a global sense, since it is valid for any admissible initial condition. In both cases, the region $\mathcal{S}_{0}$, which captures the closed-loop trajectories, contains the possible equilibrium points or limit cycles induced by the backlash operator. The equilibrium points or limit cycles induced by the saturation operator are outside the set $\mathcal{S}_{1}$.

Remark 1: In the case without backlash $(\Psi=0)$, in the definition of $M_{1}$ or $M_{2}$, one removes the columns 3 and 4 and the corresponding lines. One removes relations (14) and 


$$
M_{2}=\left(\begin{array}{cccc}
A_{0}^{\prime} P+P A_{0}+\tau_{1} P & P B-K_{0}^{\prime} T & P B K D-A_{0}^{\prime} E^{\prime} L N_{1} & -A_{0}^{\prime} E^{\prime} L N_{2} \\
\star & -2 T & -B^{\prime} E^{\prime} L N_{1}-T K D & -B^{\prime} E^{\prime} L N_{2} \\
\star & \star & -T_{3}-N_{1} L E B K D-D^{\prime} K^{\prime} B^{\prime} E^{\prime} L N_{1} & -N_{1}-D^{\prime} K^{\prime} B^{\prime} E^{\prime} L N_{2} \\
\star & \star & \star & -2 N_{2}
\end{array}\right)
$$

(16) (which, roughly speaking, corresponds to take $\tau_{1}=0$ and $\eta=1)$ and therefore one retrieves the results of [15].

Remark 2: In the case without saturation $(\varphi=0)$, in the definition of $M_{2}$ one removes the second column and line, and one sets $T=\mathbf{0}$. The results of [19] are recovered.

Remark 3: The kind of systems studied in [17] could be addressed by using the framework of the current paper. In [17], the following system is considered:

$$
\begin{aligned}
\dot{x} & =A x+B \Phi[w] \\
w & =\operatorname{sat}\left(K_{1} y+K_{2} \Phi[w]\right) \\
y & =C x+D \Phi[w]
\end{aligned}
$$

which can be rewritten, by noting $\Delta=\left(\mathbf{1}-\left(K_{1} D+\right.\right.$ $\left.\left.K_{2}\right) L\right)^{-1}$ from Assumption 2 in [17], as follows:

$$
\begin{aligned}
\dot{x}= & \left(A+B L \Delta K_{1} C\right) x+B L \Delta \varphi_{2} \\
& +B\left(\mathbf{1}+L \Delta\left(K_{1} D+K_{2}\right)\right) \Psi \\
w= & \Delta \varphi_{2}+\Delta K_{1} C x+\Delta\left(K_{1} D+K_{2}\right) \Psi
\end{aligned}
$$

with $\varphi_{2}$ defined by mimicking (5) and $\Psi$ defined in (6). According to our framework, in the global case (i.e. matrix $A$ is Hurwitz) we can relax the condition (10) in [17] linking up $K_{1}$ and $K_{2}$.

\section{NUMERICAL PROCEDURES}

The objective related to Theorem 1 is to maximize the region $\mathcal{S}_{1}$, while minimizing the region $\mathcal{S}_{0}$. This double objective needs to consider a trade-off between two conflicting goals: maximize the region of attraction of the closed loop (namely select a small trace for $\eta P$ ) and minimize the region of ultimate boundedness stability (namely select a large trace for $P$ ). Thanks to the use of a unique matrix $P$ to shape both sets $\mathcal{S}_{0}$ and $\mathcal{S}_{1}$, the objective may be expressed as maximizing the distance between those two sets, and a solution to Problem 1 is searched with the following (bilinear) optimization problem:

$\min \eta$

under conditions (12), (14), (15), (16)

In Theorem 1, relation (12) admits two kinds of nonlinearities: the products $\tau_{1} P$ and $G^{\prime} T$. These nonlinearities may be managed relatively easily. Actually, $T \in \Re^{m \times m}$ is a diagonal positive definite matrix. In the particular case of single-input systems $(m=1), T$ becomes a scalar and the optimal solution of (12) can be obtained from an iterative line search. For $m=2$, a search for the optimal solution over a bi-dimensional grid (composed by the 2 elements of $T)$ can be considered. For systems presenting high number of inputs $(m>2)$, a relaxation scheme, which translates the problem into a sequence of iterative LMI problems fixing $T$ or $G$ at each step, can be considered. The convergence of the procedure is always ensured, but not necessarily to the global optimal value. Furthermore, the convergence value depends on the initialization of $T$ or $G$ in the iterative procedure. A grid search may also be used to select $\tau_{1}$. This latter parameter being issued from the use of the $S$-procedure, its influence remains rather limited on the optimal cost of the optimization problem (27) as soon as a feasible value has been selected.

In Theorem 2, the condition (21) becomes a LMI when $\tau_{1}$ is fixed. The same treatment as in Theorem 1 may be applied.

\section{ILlustrative EXAMPLES}

Example 1: Consider first an illustrative example borrowed from [5] (Example 6) or [18] and adapted to our context (the deadzone is replaced by a backlash). This example mimics the case of a nonlinear actuator involving a backlash and a saturation (see Figure 1).

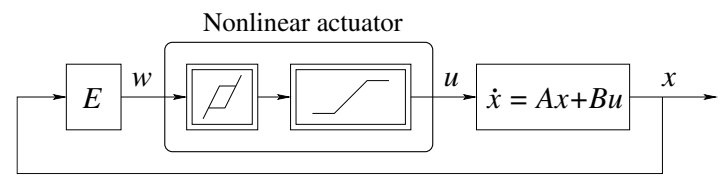

Fig. 1. Closed-loop system with backlash and saturation in the actuator

System (1) is defined by the following data:

$$
\begin{gathered}
A=\left[\begin{array}{cc}
0.5 & -1 \\
1 & 0.5
\end{array}\right] ; B=\left[\begin{array}{c}
0.5 \\
1
\end{array}\right] ; C=\left[\begin{array}{ll}
0 & 0
\end{array}\right] \\
D=1 ; E=\left[\begin{array}{ll}
0.93 & -3.84
\end{array}\right] ; K=1
\end{gathered}
$$

with $u_{0}=4, L=1$ and $\rho=0.4$.

A preliminary solution is first obtained on a rough grid then a more precise grid on a thiner interval is applied to get a solution to Problem 1. To illustrate the influence of parameter $\tau_{1}$ and matrix $T$ (actually in this example of dimension 1), the cost function $\eta$ solution to the optimization problem (27) is plotted in Figure 2.

The optimal ellipsoids $\mathcal{S}_{0}$ and $\mathcal{S}_{1}$ obtained for $\tau_{1}=0.65$ and $T=7.5$ are plotted in Figure 3 in solid lines. Trajectories starting from several initial states (for any given initial state $x(0)$ one selects $\Phi(0)=\Phi[w(0)]$ verifying (3)) are also plotted in the figure. They illustrate that the outer ellipsoid $\mathcal{S}_{1}$ obtained is a reasonable approximation of the domain of attraction. Moreover, to illustrate this aspect, the ellipsoidal approximation of the basin of attraction focusing on the case of saturation only is plotted in Figure 3 (dashed ellipsoid). It may also be compared to the outer ellipsoid corresponding to the maximal volume issued from the grid search (dashdot ellipsoid). 


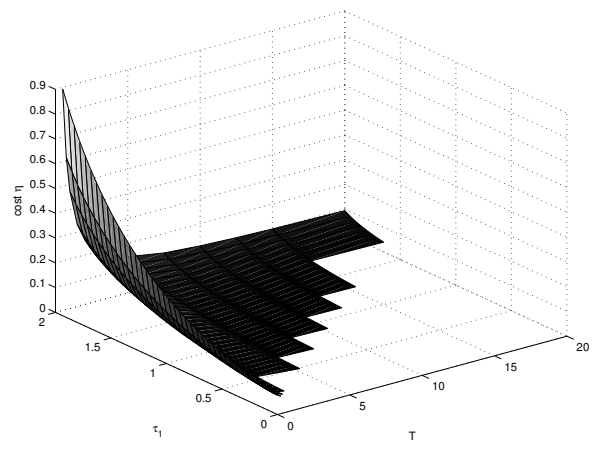

Fig. 2. Example 1 - influence of parameters $\tau_{1}$ and $T$ on the cost $\eta$

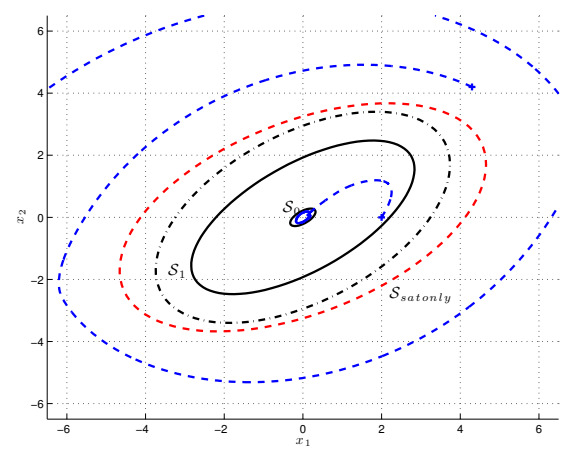

Fig. 3. Example 1 - Ellipsoids $\mathcal{S}_{0}$ and $\mathcal{S}_{1}$ corresponding to the optimal cost $\eta$ (solid lines). Ellipsoid $\mathcal{S}_{\text {satonly }}$ solution to the case with saturation element only (dashed ellipsoid, see [14]) compared to the set $\mathcal{S}_{1}$ obtained by considering the solution (on a grid search) which maximizes its volume (dashdot ellipsoid). State-space trajectories issued from $x(0)=\left[\begin{array}{ll}2 & 0\end{array}\right]^{\prime}$ (inside $\mathcal{S}_{1}$ ) and from $x(0)=\left[\begin{array}{ll}4.3 & 4.2\end{array}\right]^{\prime}$ (outside $\mathcal{S}_{1}$ ).

A zoom of the inner ellipsoid $\mathcal{S}_{0}$ and, by the way, of the state-space trajectory of the system issued from $x(0)=\left[\begin{array}{ll}20 & { }^{\prime}\end{array}\right.$ (with $\Phi(0)=1.46$ ) is plotted in Figure 4. It illustrates that the system does not converge to the origin but to a limit cycle. This limit cycle remains however confined inside the inner ellipsoid $\mathcal{S}_{0}$.

Example 2: Let us consider again the system of Example 1 but with a reorganization of the nonlinear element such as to mimic a system with input saturation and output backlash, such as given in Figure 5.

System (1) is defined by the following data:

$$
\begin{aligned}
A & =\left[\begin{array}{cc}
0.5 & -1 \\
1 & 0.5
\end{array}\right] ; B=\left[\begin{array}{c}
0.5 \\
1
\end{array}\right] ; C=\left[\begin{array}{cc}
1 & 0 \\
0 & 0
\end{array}\right] \\
D & =\left[\begin{array}{l}
0 \\
1
\end{array}\right] ; E=\left[\begin{array}{ll}
0 & 1
\end{array}\right] ; K=\left[\begin{array}{ll}
0.93 & -3.84
\end{array}\right]
\end{aligned}
$$

with the same $u_{0}=4, L=1$ and $\rho=0.4$. The linear behavior of both systems of Examples 1 and 2 is exactly the same. However, the presence of the backlash in the output makes the problem much more difficult to solve and feasibility may not be obtained depending on the organization of matrices $C, D$ and $E$. In the current case, the problem is made feasible by considering that only the second state is affected by a backlash element in its sensor.

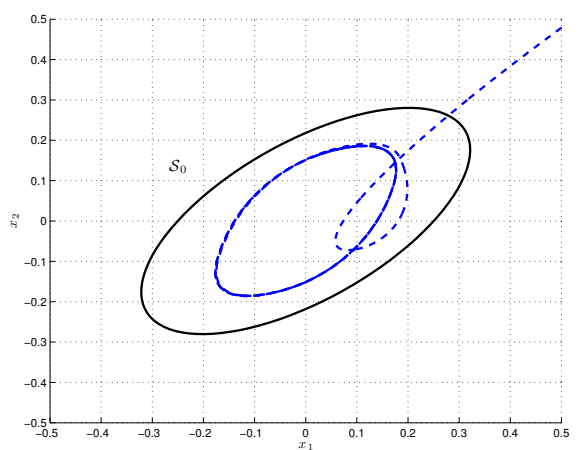

Fig. 4. Example $1-$ Zoom on the inner ellipsoid $\mathcal{S}_{0}$ and state space trajectory issued from $x(0)=\left[\begin{array}{ll}2 & 0\end{array}\right]^{\prime}$ (inside $\mathcal{S}_{1}$ )

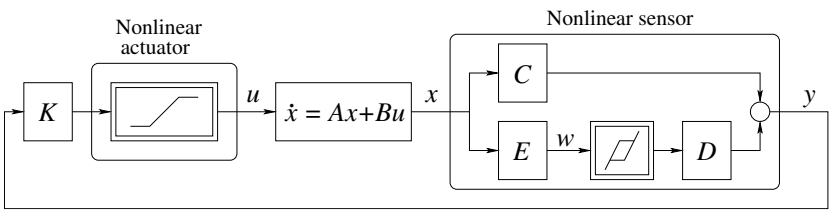

Fig. 5. Closed-loop system with backlash in the sensor and saturation in the actuator

A solution, similar to the one obtained in Example 1, is obtained for $\tau_{1}=0.65$ and $T=0.55$. The feasibility set with respect to $\tau_{1}$ and $T$ is displayed in Figure 6, so as the associated cost level $\eta$ (smallest costs in black, largest in white).

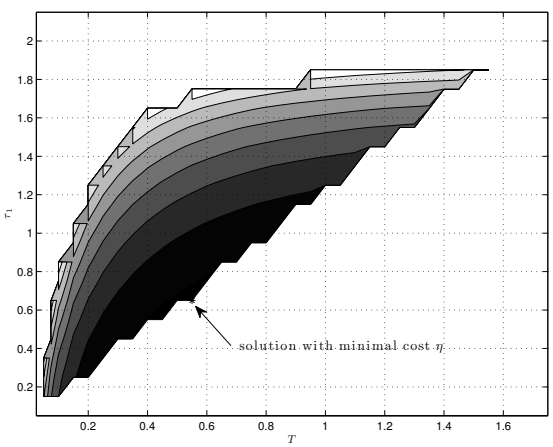

Fig. 6. Example 2 - Feasibility set of the optimization problem (27) with respect to $\tau_{1}$ and $T$ and associated cost level $\eta$. The star at $\tau_{1}=0.65$, $T=0.55$ corresponds to the optimal solution

The time evolution of $w=x_{2}$ and backlash $\Phi[w]$ is plotted in Figure 7, for the system initialized in $x(0)=$ $\left[\begin{array}{ll}1.5 & -0.9\end{array}\right]^{\prime}$ (with $\Phi(0)=-1.3$ ). It illustrates the local behavior and the limit cycle induced by the backlash.

The input signal $u$ and its value $K y$ before the saturation are plotted in Figure 8. It may verified that $u$ is initially saturated.

Example 3: Let us now consider the following system, with backlash in the output and saturation in the input:

$$
A=\left[\begin{array}{ccc}
-2 & -1 & -0.5 \\
2 & 0 & 0 \\
0 & 1 & 0
\end{array}\right] ; B=\left[\begin{array}{cc}
-0.19 & 0.41 \\
0 & -0.41 \\
0 & 0
\end{array}\right]
$$




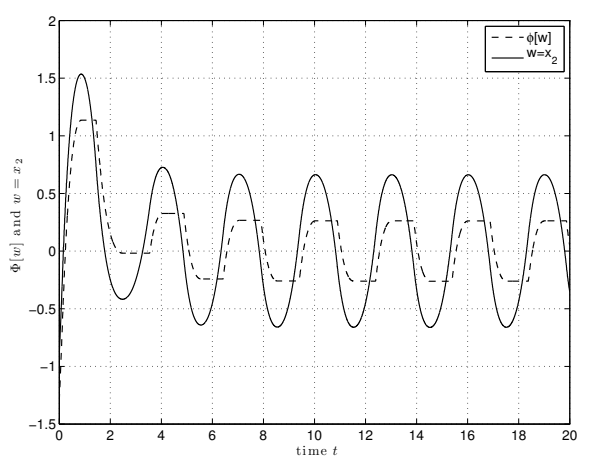

Fig. 7. Example 2 - Time-evolution of the state of $w=x_{2}$ and backlash $\Phi[w]$ for the system initialized in $x(0)=[1.5-0.9]^{\prime}$

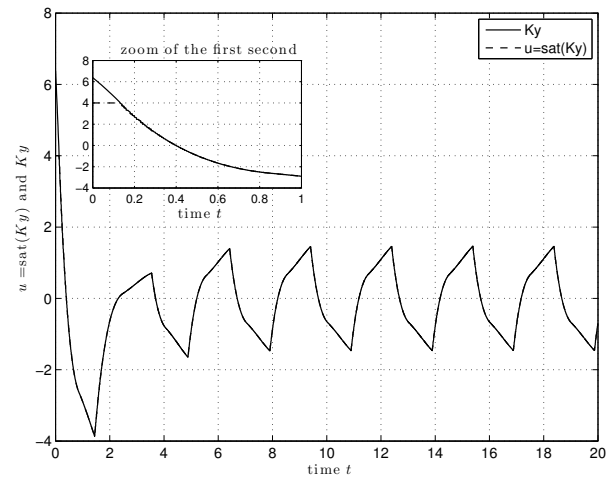

Fig. 8. Example 2 - Time-evolution of the input $u=\operatorname{sat}(K y)$ and $K y$ for the system initialized in $x(0)=[1.5-0.9]^{\prime}$ and zoom during the first second

$C=\mathbf{0} ; \quad D=\mathbf{1} ; E=\mathbf{1} ; K=\left[\begin{array}{ccc}1.875 & -0.188 & 0.094 \\ 1 & 0.75 & 1\end{array}\right]$

The open-loop matrix is Hurwitz and Theorem 2 may be applied to solve the global case described in Problem 2. The ellipsoid $\mathcal{S}_{0}$ solution to the optimization problem (27) with $\tau_{1}=0.3$ selected with a grid search is plotted in Figure 9. Several trajectories are also plotted which enter the ellipsoid and, then, remain inside.

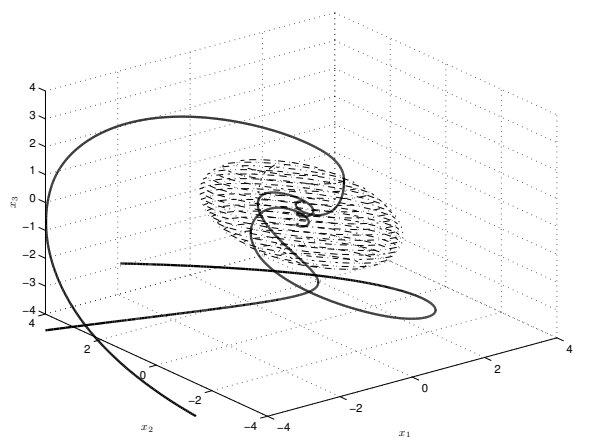

Fig. 9. Example 3 - Ellipsoidal set $\mathcal{S}_{0}$ and several trajectories of the closed-loop system

\section{CONCLUDING REMARKS}

Systems presenting a backlash in the loop and a saturated input have been considered in this paper. The stability has been studied for this class of nonlinear systems by exploiting generalized sector conditions of both nonlinearities. By means of a Lyapunov function, some numerically tractable sufficient conditions have been derived for the regional (local) or global stability of a neighborhood of the origin (where the solutions are captured). The main results have been illustrated with different examples, in particular for systems with saturation in the input and backlash in the output. Several generalizations may be considered. Following the research line suggested in this paper, the design of antiwindup compensator using the knowledge on the backlash operator could be pursued in order to alleviate the bad effects due to both saturation and backlash operators.

\section{REFERENCES}

[1] A. Barreiro and A. Baños. Input-output stability of systems with backlash. Automatica, 42(6):1017-1024, 2006.

[2] M. Brokate and J. Sprekels. Hysteresis and phase transitions, volume 121 of Applied Mathematical Sciences. Springer-Verlag, New York, 1996.

[3] M. L. Corradini and G. Orlando. Robust stabilization of nonlinear uncertain plants with backlash or dead zone in the actuator. IEEE Transactions on Control Systems Technology, 10(1):158 - 166, 2002.

[4] M del Carmen Rodriguez and W.P. Heath. Controller structure for plants with combined stauration and deadzone/backlash. In International Conference on Control Application (CCA), pages 1394-1399, Dubrovnik, Croatia, October 2012.

[5] I-K. Fong and C-C. Hsu. State feedback stabilization of single input systems through actuators with saturation and deadzone characteristics. In Conference on Decision and Control, Sydney, Australia, 2000.

[6] T. Hu and Z. Lin. Control systems with actuator stauration: Analysis and design. Birkhauser, Boston, 2001.

[7] B. Jayawardhana, H. Logemann, and E.P. Ryan. The circle criterion and input-to-state stability. IEEE Control Systems Magazine, 31(4):3267, 2011.

[8] V. Kapila and K. Grigoriadis (Eds.). Actuator saturation control. Marcel Dekker, Inc., 2002.

[9] H.K. Khalil. Nonlinear Systems. Prentice-Hall, 3rd edition, 2002.

[10] J. W. Macki, P. Nistri, and P. Zecca. Mathematical models for hysteresis. SIAM Review, 35(1):94-123, 1993.

[11] M. Nordin, X. Ma, and P.O. Gutman. Controlling mechanical systems with backlash: a survey. Automatica, 38:1633-1649, 2002.

[12] T. Paré, A. Hassibi, and J. How. A KYP lemma and invariance principle for systems with multiple hysteresis non-linearities. Int. J. of Control, 74(11):1140-1157, 2001.

[13] P.G. Park, D. Banjerdpongchai, and T. Kailath. The asymptotic stability of nonlinear (Lur'e) systems with multiple slope restrictions. IEEE Transactions on Automatic Control, 43(7):979-982, 1998.

[14] S. Tarbouriech, G. Garcia, J.M. Gomes da Silva Jr., and I. Queinnec. Stability and stabilization of linear systems with saturating actuators. Springer, London, 2011.

[15] S. Tarbouriech, C. Prieur, and J.M. Gomes da Silva Jr. Stability analysis and stabilization of systems presenting nested saturations. IEEE Transactions on Automatic Control, 51(8):1364-1371, 2006.

[16] S. Tarbouriech, C. Prieur, and I. Queinnec. Stability analysis for linear systems with input backlash through sufficient LMI conditions. Automatica, 46(11):1911-1915, 2010.

[17] S. Tarbouriech, C. Prieur, I. Queinnec, and T. Simões dos Santos. Global stability for systems with nested backlash and saturation operators. In American Control Conference, Baltimore, USA, July 2010.

[18] S. Tarbouriech, I. Queinnec, T. Alamo, M. Fiacchini, and E. F. Camacho. Ultimate bounded stability and stabilization of linear systems interconnected with generalized saturated functions. Automatica, 47:1473-1481, 2011.

[19] S. Tarbouriech, I. Queinnec, and C. Prieur. Stability analysis and stabilization of systems with input backlash. IEEE Transactions on Automatic Control, to appear.

[20] A. Taware and G. Tao. Control of sandwich nonlinear systems. Lecture Notes in Control and Information Sciences, vol.288, Springer-Verlag, Berlin, 2003. 